\title{
Poly ( $\varepsilon$-caprolactone) nanofibrous ring surrounding a polyvinyl alcohol hydrogel for the development of a biocompatible two-part artificial cornea
}

\author{
This article was published in the following Dove Press journal: \\ International Journal of Nanomedicine \\ 13 July 201 I \\ Number of times this article has been viewed
}

\author{
Haleh Bakhshandeh' \\ Masoud Soleimani ${ }^{2}$ \\ Saied Shah Hosseini ${ }^{3}$ \\ Hassan Hashemi ${ }^{3}$ \\ Iman Shabani ${ }^{4}$ \\ Abbas Shafiee ${ }^{5}$ \\ Amir Houshang Behesht \\ Nejad ${ }^{6}$ \\ Mohammad Erfan' \\ Rassoul Dinarvand ${ }^{7}$ \\ Fatemeh Atyabi ${ }^{7}$ \\ 'Department of Pharmaceutics, \\ School of Pharmacy, Shaheed \\ Beheshti Medical University, Tehran, \\ Iran; ${ }^{2} \mathrm{Hematology}$ Department, \\ Faculty of Medical Science, Tarbiat \\ Modares University, Tehran, Iran; \\ ${ }^{3}$ Noor Ophthalmology Research \\ Center, Noor Eye Hospital, Tehran, \\ Iran; ${ }^{4}$ Nanotechnology and Tissue \\ Engineering Department, Stem Cell \\ Technology Research Center, Tehran, \\ Iran; ${ }^{5}$ Stem Cell Biology Department \\ Stem Cell Technology Research \\ Center, Tehran, Iran; ${ }^{6}$ Ophthalmology \\ Department, Tehran University \\ of Medical Sciences, Tehran, Iran; \\ ${ }^{7}$ Nanotechnology Research Center, \\ Faculty of Pharmacy, Tehran University \\ of Medical Sciences, Tehran, Iran
}

Correspondence: Fatemeh Atyabi Nanotechnology Research Center, Faculty of Pharmacy, Tehran University of Medical Sciences, Tehran, 14174, Iran Tel +982166959052

Fax +98 21 66959052

Email atyabifa@tums.ac.ir

\begin{abstract}
The study aimed to fabricate and characterize a 2-part artificial cornea as a substitute for penetrating keratoplasty in patients with corneal blindness. The peripheral part of the artificial cornea consisted of plasma-treated electrospun poly ( $\varepsilon$-caprolactone) (PCL) nanofibers, which were attached to a hydrogel disc of polyvinyl alcohol (PVA) as a central optical part. The physical properties of the prepared artificial cornea, including morphology, mechanical properties, light transmittance, and contact angle, were assessed. Cell attachment and proliferation studies were performed on rabbit limbal stem cells. The SEM image of the polymeric system showed that the peripheral part formed a highly porous scaffold that could facilitate tissue biointegration. Assessment of the mechanical properties of the peripheral nanofibrous part and the hydrogel optical part showed suitable elasticity. Young's modulus values of the electrospun PCL skirt and PVA hydrogel core were 7.5 and 5.3 MPa, respectively, which is in line with the elasticity range of natural human cornea $(0.3-7 \mathrm{MPa})$. The light transmittance of the central part was $>85 \%$ when measured in the 400-800 $\mathrm{nm}$ wavelength range. The plasma-treated PCL nanofibrous scaffold promoted limbal stem cell adhesion and proliferation within 10 days. These results confirmed that the polymeric artificial cornea showed suitable physical properties and good biocompatibility and epithelialization ability.
\end{abstract}

Keywords: two part artificial cornea, nanofibers, electrospun, poly ( $\varepsilon$-caprolactone), polyvinyl alcohol hydrogel, limbal stem cells

\section{Introduction}

The cornea is the first and most important refractive layer of the eye whose function is to focus light onto the retina. It also functions as a physical and optical barrier that protects the intraocular part of the eye by absorbing UV radiation and preventing the invasion of microbes and other particles. ${ }^{1-5}$

Corneal blindness is a major and widespread eye problem, with approximately 10 million people worldwide who are blind because of corneal disorders. ${ }^{2,3,6-10}$ Injury to the cornea can be a consequence of trauma, burns, infections, disease scars, and previous surgery resulting in inflammation, vascularization, scar formation, opacification, and finally blindness. . $^{1,6,11}$

The current treatment of corneal blindness is penetrating keratoplasty or corneal transplantation. Although the success rate of corneal transplantation is higher than that of other tissue transplants, certain issues such as tissue availability, cost, risk of infection, potential complications (astigmatism, glaucoma, keratoconus), recurrent corneal graft 
rejection, and cases of chemical burn or inactive central scars can limit the use of keratoplasty. Therefore, the development of an "artificial cornea" (keratoprosthesis, KPro) is a desirable option to overcome these problems., , $3,5,8-12^{-12}$

An artificial cornea generally consists of an optical central part or "core" that should be transparent, and a biocompatible peripheral part or "skirt" that should allow for cellular integration. ${ }^{13}$ The main problem of KPro was, and still is, the rejection of the prosthesis or "extrusion" because of erosive tissue necrosis (melting) around the margin of the prosthesis. $^{1,10}$

The first keratoprostheses were made from glass, crystal, and celluloid implants in the 19th century. Because of poor results and the success of human to human corneal transplantation, the interest in artificial corneas decreased. In 1950, polymethyl methacrylate (PMMA), as a synthetic polymer with low toxicity and good optical properties, was applied for the preparation of an artificial cornea. Studies showed that PMMA could not be maintained in the eye for a long period as a result of its rigidity and hydrophobicity. ${ }^{6,10}$ Since the 1990 s, a new generation of experimental KPros have been developed to solve the complications associated with the existing prostheses by increasing their hydrophilicity and flexibility to stimulate biocolonization of host cells on the peripheral part of the prosthesis, and consequently improve their biocompatibility. ${ }^{5,9}$ Chirila KPro or AlphaCor ${ }^{\mathrm{TM}}$ (Addition Technology Inc, Des Plaines, IL), one of the recent Food and Drug Administration (FDA) -approved keratoprostheses, is an artificial device consisting of a homogeneous spongy peripheral skirt and a transparent central part. Both parts are made from poly-2-hydroxyethyl methacrylate. ${ }^{14,15}$ However, the development of inexpensive and biocompatible materials that can be manufactured using novel and simple techniques is necessary to further improve the biocompatibility of artificial corneas.

Biocompatibility depends on the chemical properties (eg, hydrophilicity) of the polymers used, in particular those forming the peripheral part, and the architecture of the prosthesis (eg, porous structure, size, and number of pores). These properties induce cell attachment by improving the hydrophilicity of the corneal matrix and the circulation of nutrient fluids and gases.

Ideally, the peripheral part of a prosthesis should mimic the structure and biological function of the native extracellular matrix (ECM) of the surrounding tissue. The ECM is composed of proteoglycans (ground substances) and collagens (fibrous proteins). The collagen fibrous structure is arranged in a 3-dimensional nanofiber network. ${ }^{16}$ Therefore, in the present study, the peripheral part of the KPro consisted of poly ( $\varepsilon$-caprolactone) (PCL) nanofibers that were manufactured using an electrospinning process.

Electrospinning is a novel method for the fabrication of unwoven, porous, and 3-dimensional polymeric nanofibrous scaffolds. In this method, ultrafine fibers are produced by electrically charging a suspended droplet of polymer solution. The nanofibrous structure made by electrospinning has a high surface area to volume ratio, which provides an increased surface for cell adhesion in comparison with other structures. ${ }^{16-19}$ In addition, simplicity and cost effectiveness are 2 attractive features of electrospinning. ${ }^{18}$

PCL is a biocompatible, biodegradable, and inexpensive semicrystalline aliphatic polymer that has been used in an electrospun form. Furthermore, this material is compatible with various types of cells. In addition, the slow degradation rate of PCL makes it suitable for application in the peripheral part of an endurable KPro. ${ }^{18-21}$ The application of certain surface treatment methods such as plasma treatment can increase the surface hydrophilicity of these polymeric nanofibers. Plasma treatment is one of the best techniques for the development of surface hydrophilicity, which can improve cell attachment, expansion, and proliferation of host cells on polymeric surfaces. ${ }^{17,22-24}$

Polyvinyl alcohol (PVA) is a good candidate material for the optical part of 2-part (core-and-skirt) artificial corneas. PVA is a representative water-soluble, biocompatible and processable polymer. Physically cross-linked PVA made by a freeze-thawing method under specific conditions has excellent light transparency, nutrition permeability, and suitable mechanical properties, which are necessary for keratoprosthesis. ${ }^{12,25,26}$

In this study, we prepared and characterized a biocompatible 2-part artificial cornea with a transparent PVA hydrogel core surrounded by a porous plasma-treated electrospun PCL skirt.

\section{Materials and methods Materials}

PCL with $\mathrm{M}_{\mathrm{n}} \sim 80,000 \mathrm{Da}$ was purchased from Sigma-Aldrich (St. Louis, MO). The solvents $N, N$-dimethylformamide (DMF) and dimethyl sulfoxide (DMSO) were obtained from Merck (Darmstadt, Germany). PVA with $M_{w} \sim 200,000$ Da was purchased from Merck (Darmstadt, Germany). The other chemicals were obtained from Sigma-Aldrich. 


\section{Fabrication of the PCL electrospun nanofibrous scaffold (skirt)}

PCL nanofibers were produced by an electrospinning method. ${ }^{17}$ Briefly, a PCL solution ( $5 \%$ by weight) was prepared in chloroform and DMF in a 8.5:1.5 ratio and fed into a blunted needle with a syringe pump. A rotating cylindrical drum was used as a collector and placed at a distance of $15 \mathrm{~cm}$ from the needle. Voltage was $20 \mathrm{kV}$ and flow rate was $0.5 \mathrm{~mL} / \mathrm{h}$.

To increase the hydrophilicity of the nanofibers, surface modification of the PCL scaffold was performed by plasma treatment.

A low-frequency plasma generator ( $40 \mathrm{kHz}$ frequency) with a cylindrical quartz reactor (Diener Electronics, Ebhausen, Germany) was used. Pure oxygen gas was introduced into the reaction chamber at a pressure of 0.4 mbar, and the glow discharge was ignited for 3 minutes.

Plasma-treated sheets were cut into disks of $1 \mathrm{~cm}$ diameter and again punched with a device of $0.5 \mathrm{~cm}$ diameter to make a rim $0.5 \mathrm{~cm}$ wide as the peripheral part (skirt) of the KPro.

\section{Preparation of the PVA hydrogel (core) and attachment to the PCL nanofibrous skirt}

A $15 \%$ by weight PVA solution was prepared by mixing the polymer in deionized water and DMSO $(2: 8, \mathrm{v}: \mathrm{v})$. The mixture was placed in a loosely capped container. The PVA solution was obtained upon heating the mixture for 2 hours in a $140^{\circ} \mathrm{C}$ oven. The mixture was then transferred to a sterile ventilation hood and stirred to ensure a homogenous solution. ${ }^{27}$ When the solution cooled to $80-100^{\circ} \mathrm{C}$, a suitable volume was poured into the center of a circular mold to prepare a disk with a diameter slightly larger than $0.5 \mathrm{~cm}$ and about $0.5 \mathrm{~mm}$ thick. After a few seconds, the peripheral part was thermally attached to the core. The mold was then subjected to 5 cycles of freezing and thawing. The sample was allowed to cool for about 24 hours for each freeze-thaw cycle.

\section{Characterization of physical properties} Mechanical properties

Plasma-treated PCL nanofiber and PVA hydrogel sheets approximately $0.5 \mathrm{~mm}$ thick were cut into $1 \times 5 \mathrm{~cm}^{2}$ rectangular shapes. The tensile properties of the samples were determined using the Universal Testing Machine (STM-20;
SANTAM, Tehran, Iran). Samples were vertically mounted on 2 mechanical gripping units of the tensile tester at their ends, leaving a $3 \mathrm{~cm}$ gauge length for mechanical loading. Load-deformation data were recorded at a deforming speed of $50 \mathrm{~mm} / \mathrm{min}$ and the stress-strain curves of 2 structures were constructed from the data.

\section{Transparency measurement}

PVA sheets of $0.5 \mathrm{~mm}$ thickness were evaluated for transparency by scanning at the visible wavelength range (400-800 nm) with a spectrophotometer (CECIL-CE 7500; Cecil instruments, Cambridge, UK) in wet and dry forms. In the wet form, the PVA sheet was floated in a cuvette containing water, and for preparation of the dry sample, the PVA sheet was placed at room temperature $\left(21^{\circ} \mathrm{C}\right)$ in $70 \%$ relative humidity for 6 hours.

\section{Morphology study}

The morphology of the PCL nanofibers and PVA hydrogel was studied using a scanning electron microscope (SEM; XL30; Philips, Eindhoven, Netherlands). Samples were coated with gold by a sputter coater, and the diameter of the fibers was determined from images by using an image analysis software.

\section{Contact angle measurement}

The wettability of the nanofibrous skirt after surface modification was assessed by the sessile drop method with a G10 Krüss contact angle goniometer (Krüss, Hamburg, Germany), at room temperature. A water droplet was placed on the sample surface, and the contact angle was measured after 10 seconds.

\section{Cell culture study}

Rabbit limbal stem cell isolation and expansion

Limbal biopsy specimens were harvested from healthy New Zealand rabbits according to the ARVO Statement for the use of Animals in Vision and Ophthalmic Research. Specimens were digested by $2.4 \mathrm{IU} / \mathrm{mL}$ dispase and collagenase in DMEM for 3-4 hours in a $\mathrm{CO}_{2}$ incubator. Cells were then collected by centrifugation at $1500 \mathrm{~g}$ for 5 minutes and cultured for 7-10 days in 6-well plastic plates coated with $1 \%$ gelatin. The limbal stem cells (LSCs) were grown in DMEM/F12 supplemented with insulin $(5 \mu \mathrm{g} / \mathrm{mL})$, epidermal growth factor $(10 \mathrm{ng} / \mathrm{mL})$, hydrocortisone $(0.4 \mu \mathrm{g} / \mathrm{mL})$, and fetal bovine serum (FBS) (7\%). 


\section{Cell attachment study}

For the cell attachment study, LSCs were seeded onto the $1.5 \mathrm{~cm}$ disk of the plasma-treated PCL scaffold at a density of $10^{4} \mathrm{~cm}^{-1}$ and incubated in the culture medium. After 2 days, the sample was fixed for 1 hour with $2.5 \%$ glutaraldehyde. After the incubation period, the sample was rinsed with phosphatebuffered saline (PBS), then gradually dehydrated with an ethanol gradient $(60 \%, 70 \%, 80 \%, 90 \%$, and $100 \%)$ for at least 5 min at each concentration. Thereafter, SEM photography and 4',6-diamidino-2-phenylindole (DAPI) staining of the prepared samples were performed to analyze cell attachment.

\section{Cell proliferation study}

For the cell proliferation assay, $5 \times 10^{3}$ LSCs were seeded on a PCL nanofibrous scaffold and on tissue culture polystyrene (TCPS) as controls.

The cellular constructs and controls were maintained in an incubator at $37^{\circ} \mathrm{C}$, and the cell culture medium was changed every 3 days. After 1, 3, 5, 7, and 10 days, samples were harvested, washed with PBS to remove nonadherent cells, and exposed to the indicated amount of 3-(4,5-dimethylthiazol-2-yl)-2,5-diphenyltetrazolium bromide (MTT; Sigma-Aldrich. After 4 hours of incubation, $200 \mu \mathrm{L}$ of DMSO was added to each well and the optical density was read in a microplate reader (Anthos 2010; Biochrom, Cambridge, UK) at $570 \mathrm{~nm}$.

\section{Results}

\section{Characterization of physical properties Mechanical properties}

The mechanical properties of electrospun nanofibrous and hydrogel sheets were determined separately using the Universal Testing Machine. A representative stress-elongation percentage (\%) curve is shown in Figure 1. The Young's modulus (mean E), break stress, and break force of both structures are shown in Table 1.

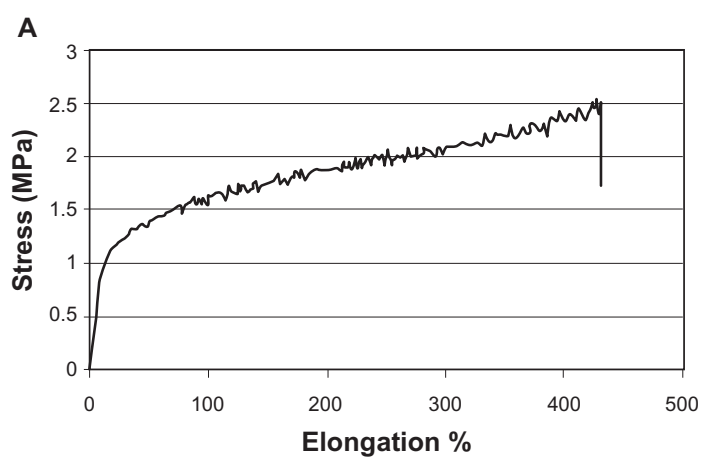

Figure I Stress-strain curve, PCL nanofibrous matrix (A), PVA hydrogel (B). Abbreviations: PCL, poly ( $\varepsilon$-caprolactone); PVA, polyvinyl alcohol.
Table I Tensile property of polymers

\begin{tabular}{lll}
\hline & $\begin{array}{l}\text { PCL nanofibrous } \\
\text { matrix }\end{array}$ & PVA hydrogel \\
\hline Tensile modulus (MPa) & 7.5 & 5.3 \\
Ultimate tensile stress (MPa) & $2.53 \pm 0.58$ & $0.85 \pm 0.55$ \\
Ultimate tensile strain (\%) & $427.2 \pm 24.6$ & $284.8 \pm 48.6$ \\
\hline
\end{tabular}

Abbreviations: PCL, poly ( $\varepsilon$-caprolactone); PVA, polyvinyl alcohol.

\section{Transparency measurement}

Figure 2 shows the visible light transmittance of the PVA samples as the optical part of the KPro in dry and wet form. The prepared samples showed a light transmittance higher than $85 \%$ in the $400-800 \mathrm{~nm}$ wavelength range.

\section{Morphology study}

SEM micrographs of the electrospun nanofibrous skirt and hydrogel core (Figure 3 ) revealed that the PCL nanofibrous matrix has a very porous structure. Homogeneously distributed pores were interconnected with an average diameter of 10-30 $\mu \mathrm{m}$, while the PVA core did not contain pores.

\section{Contact angle measurement}

To compare the hydrophilicity of the surface of PCL scaffolds, the water contact angle was measured before and after surface modification. The water contact angles of PCL and plasma-treated PCL were 131.5 and 20 degrees, respectively.

\section{Cell culture study}

\section{Cell attachment study}

DAPI staining and SEM showed the attachment of LSCs on plasma-treated PCL nanofibers after 3 days (Figure 4).

Identification of LSCs was confirmed by their morphology, and the expression of some epithelial markers (K3, K12, P63) was analyzed by reverse transcription polymerase chain reaction (data not shown).

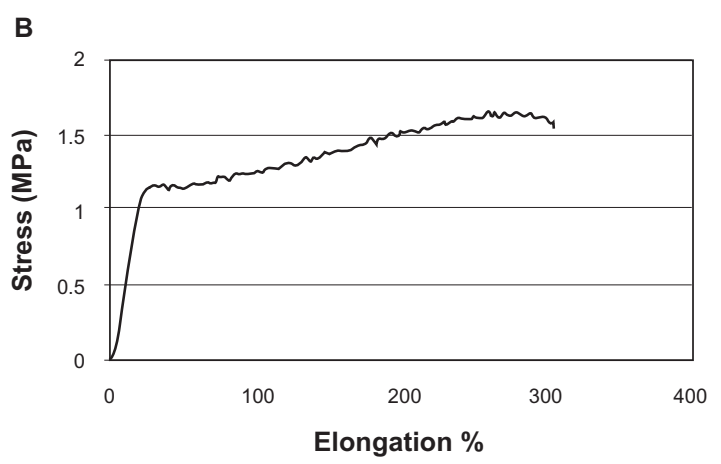




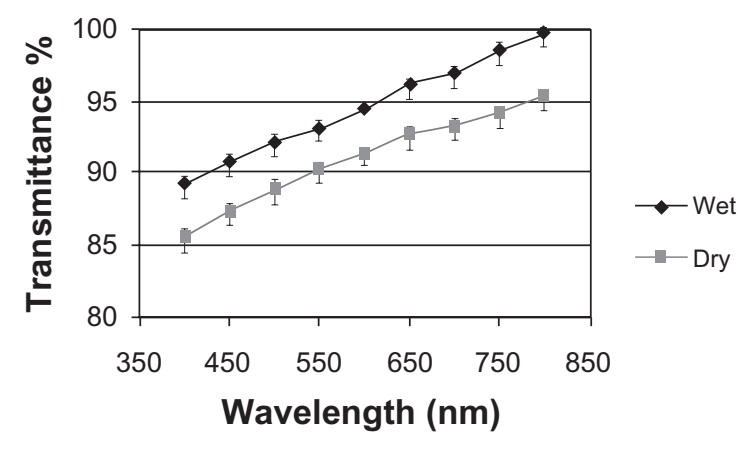

Figure 2 Light transmittance of wet and dry PVA hydrogel. Abbreviation: PVA, polyvinyl alcohol.

\section{Cell proliferation study}

A cell proliferation assay was performed to evaluate cell growth on the nanofibrous skirt and for the assessment of biocompatibility. Figure 5 clearly shows a gradual increase in the proliferation rate of LSCs on the scaffold surface over a 10-day period.

\section{Discussion}

The use of an artificial cornea for the treatment of corneal disorders and blindness is a very attractive concept. The artificial cornea as a substitute for keratoplasty has many advantages, among which the cost effectiveness and feasibility are important. Although several models of KPros have been designed for clinical use, few models have received FDA approval and are currently on the market. ${ }^{15,28}$ The most critical problem of clinically approved KPros is bioincompatibility, followed by extrusion, glucoma, and endophthalmitis. Improvements in the design and materials of KPros are therefore necessary to increase tissue biointegration and biocompatibility and to prevent extrusion. A biocompatible artificial cornea showing suitable tissue biointegration and adequate optical and mechanical properties can be a substitute for damaged corneas.

The mechanical properties of an artificial cornea are important aspects to be considered in the design of a scaffold. The preparation of a nanofibrous scaffold as a skirt of an artificial cornea is not only meant to facilitate host cell residence and spreading but also for the maintenance of mechanical stability at the implantation site. This structure must retain its integrity and stability through surgery and the physician's handling. ${ }^{16}$ Evaluation of the mechanical properties of the present artificial cornea showed that the core and skirt had a Young's modulus (an indicator of elasticity) of 7.5 and $5.3 \mathrm{MPa}$, respectively, which falls within the range measured in the human cornea $(0.3-7 \mathrm{MPa}){ }^{2,29}$ These results show that the combined structure should have the capacity to tolerate incoming pressure. In addition, the thermal attachment of the 2 parts achieved by the interation of the hydrogel into nanofibers results in a tight interface that is completely resistant to stresses in this region.

Transparency is one of the most important properties of an artificial cornea. The measurement of the optical properties of the hydrogel core of our fabricated KPro showed that this structure has an excellent light transmittance.

As shown in the SEM micrographs, the PVA hydrogel did not appear to have any pores. Although the use of a high percentage of DMSO in the solvent mixture for the preparation of PVA hydrogels results in smaller and more homogenous pores, the absence of pores in our hydrogel was likely caused by the sample drying out and the consequent collapse of the pore structure during the SEM process. The PCL nanofibrous structure is highly porous. High porosity provides increased space for cell settling and facilitates the efficient exchange of metabolic waste and nutrients between the matrix and the environment. These characteristics are principal criteria for successful tissue biointegration. ${ }^{16,30}$

Moreover, when nanofibers are generated in an electrostatic field, they are randomly deposited on the collector layer by layer with loose junctions, and various diameters of pores are created. Cells can force their way into pores with their amoeboid movement. This dynamic structure provides cells with the opportunity to adjust to the optimal pore diameter and grow into the mat even though some pores are relatively
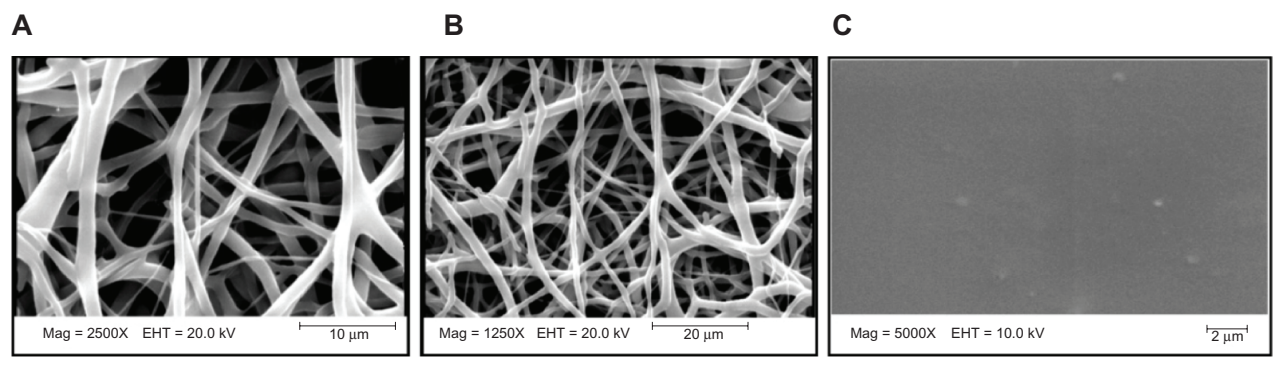

Figure 3 SEM micrographs of PCL nanofibers (A, B) and PVA hydrogel (C). Abbreviations: PCL, poly ( $\varepsilon$-caprolactone); PVA, polyvinyl alcohol. 
A

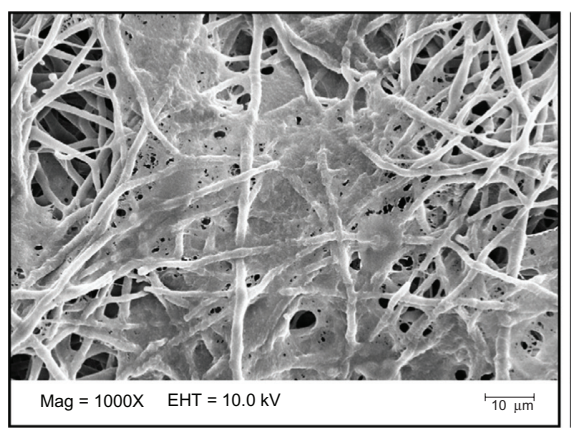

B

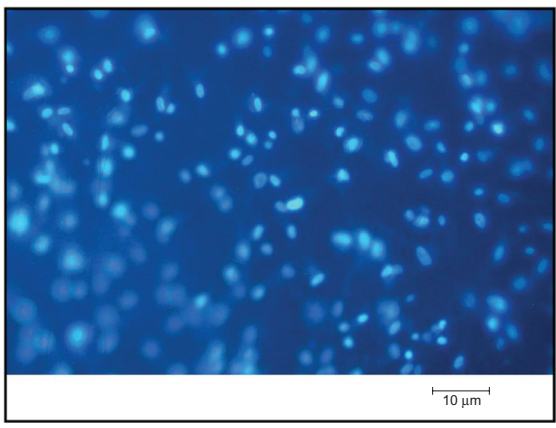

Figure 4 SEM micrograph (A) and DAPI staining image (B) of LSC attachment onto the surface of plasma-treated PCL nanofibers after 3 days. Abbreviations: DAPI, 4',6-diamidino-2-phenylindole; LSC, limbal stem cell; PCL, poly ( $\varepsilon$-caprolactone).

small. ${ }^{16}$ This phenomenon can help promote cellular invasion into the prosthesis.

As mentioned before, the surface of the PCL nanofibrous mat was modified by plasma treatment. Many studies have revealed that cell attachment and proliferation on the surface of biomaterials increases when surface hydrophilicity is increased. ${ }^{17,23,31,32}$

To compare the hydrophilicity of the surface of the PCL scaffold before and after surface modifications, the water contact angle of the specimens was measured. The results showed that the hydrophilicity of the surfaces was increased after surface modification, confirming the role of hydrophilic groups $(\mathrm{OH}$ and $\mathrm{COOH})$ in increasing the hydrophilicity of surfaces after plasma treatment. Plasma surface treatment of a polymeric matrix with $\mathrm{N}_{2}, \mathrm{O}_{2}$, and $\mathrm{NH}_{3}$ makes the surface of the matrix more hydrophilic and more bioadhesive. ${ }^{23,31}$ The SEM images of the attachment of cells to the nanofibrous structure show significant expansion of the cells on plasma-treated surfaces, thus confirming their biocompatibility and tissue biocolonization. The molecular structure of adhesive molecules in the cell membrane promotes better and more stable attachment to hydrophilic surfaces in comparison with hydrophobic ones. ${ }^{17,31}$

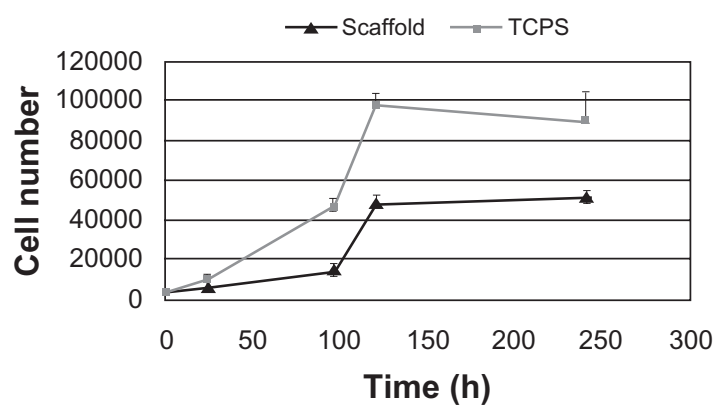

Figure 5 Proliferation of LSCs seeded on plasma-treated PCL matrix and TCPS. Abbreviations: LSCs, limbal stem cells PCL, poly ( $\varepsilon$-caprolactone); TCPS, tissue culture polystyrene.
In the present study, cell attachment and proliferation studies confirmed the considerable adhesion and expansion of epithelial cells onto the plasma-treated PCL nanofibrous skirt. Rabbit limbal cells are epithelial stem cells selected based on the evaluation of biocompatibility with this prosthesis. The attachment of these cells, as well as the rate of cell proliferation, can be used as criteria to evaluate the epithelialization ability of this corneal implant.

Results of the cell proliferation assay revealed that the electrospun nanofibrous structure promoted cell growth. Although cell proliferation onto TCPS was higher than in the scaffold, cell proliferation onto the scaffold up to day 10 showed an increasing trend. Moreover, on day 10, cell proliferation on TCPS decreased while that on the nanofibrous scaffold increased compared with day 5 . This novel structure provides an increased surface area for cells to attach, owing to its 3-dimensional feature and its high surface area to volume ratio. ${ }^{16}$

Although the PVA hydrogel has a low affinity for cell adhesion, its hydrophilicity and biocompatibility is sufficient to allow spreading of epithelial cells that have attached and proliferated onto the margin. However, future studies should evaluate the effects of immobilizing structural and adhesive biomolecules such as collagen, laminin, or fibronectin to improve the epithelialization ability of this structure.

\section{Acknowledgment}

This project was supported financially by deputy for research and technology of the Ministry of Health and Medical Education of Iran.

\section{Disclosure}

The authors declare no conflicts of interest in relation to this work. 


\section{References}

1. Noolandi J, Ta C, Huie JP, et al. inventors; The Board of Trustees of the Leland Stanford Junior University, assignee. Artificial Cornea. United States patent US 6976997. Dec 20, 2005.

2. Duan X, Sheardown H. Dendrimer crosslinked collagen as a corneal tissue engineering scaffold: mechanical properties and corneal epithelial cell interactions. Biomaterials. 2006;27:4608-4617.

3. Li F, Carlsson D, Lohmann C, et al. Cellular and nerve regeneration within a biosynthetic extracellular matrix for corneal transplantation. Am J Ophthalmol. 2004;138:318.

4. Chirila TV, Hicks CR, Dalton PD, et al. Artificial cornea. Prog Polym Sci. 1998;23:447-473.

5. Albon J. Corneal transplantation and artificial cornea. J Mech Med Biol. 2003;3:95-106.

6. Darougar S, Darougar D, inventors; Nields and Lemack, assignee. Artificial Cornea. United States patent US 20070168025. Jul 19, 2007.

7. Carlsson DJ, Li F, Shimmura S, Griffith M. Bioengineered corneas: how close are we? Curr Opin Ophthalmol. 2003;14:192-197.

8. Myung D, Ta C, Farooqui N, et al, inventors; Lumen Intellectual Property Services, Inc., assignee. Artificial cornea. United States patent US 20060287721. Dec 21, 2006.

9. Hicks C, Crawford G, Chirila T, et al. Development and clinical assessment of an artificial cornea. Prog Retin Eye Res. 2000;19:149-170.

10. Chirila TV. An overview of the development of artificial corneas with porous skirts and the use of PHEMA for such an application. Biomaterials. 2001;22:3311-3317.

11. Crawford GJ, Hicks CR, Lou XP, et al. The Chirila Keratoprosthesis: phase I human clinical trial *1. Ophthalmology. 2002;109:883.

12. Miyashita H, Shimmura S, Kobayashi H, et al. Collagen-immobilized poly(vinyl alcohol) as an artificial cornea scaffold that supports a stratified corneal epithelium. J Biomed Mater Res B Appl Biomater. 2006;76: 56-63.

13. Yoshito I, Junichi O, Naoki K, et al., inventors; Menicon Co., Ltd., assignee. Artificial Cornea. United States patent US 6391055. May 21, 2002.

14. Chirila TV, Constable IJ, Crawford GJ, et al. Poly(2-hydroxyethyl methacrylate) sponges as implant materials: in vivo and in vitro evaluation of cellular invasion. Biomaterials. 1993;14:26-38.

15. Holak S, Holak H, Bleckmann H. AlphaCor ${ }^{\mathrm{TM}}$ keratoprosthesis: postoperative development of six patients. Graefes Arch Clin Exp Ophthalmol. 2009;247:535-539.

16. Li WJ, Laurencin CT, Caterson EJ, Tuan RS. Electrospun nanofibrous structure: A novel scaffold for tissue engineering. J Biomed Mater Res. 2002;60:613-621.

17. Shabani I, Haddadi-Asl V, Seyedjafari E, Babaeijandaghic F, Soleimani M. Improved infiltration of stem cells on electrospun nanofibers Biochem Biophys Res Commun. 2009;382:129-133.
18. Ghasemi-Mobarakeh L, Morshed M, Karbalaie K, Fesharaki M. Electrospun poly ( $\varepsilon$-caprolactone) nanofiber mat as extracellular matrix. Yakhteh Medical Journal. 2008;10:179-184.

19. Luong-Van E, Grn̈dahl L, Chua KN, Leong KW, Nurcombe V, Cool SM. Controlled release of heparin from poly([epsilon]-caprolactone) electrospun fibers. Biomaterials. 2006;27:2042-2050.

20. Meng ZX, Zheng W, Li L, Zheng YF. Fabrication and characterization of three-dimensional nanofiber membrance of PCL-MWCNTs by electrospinning. Mater Sci Eng C Mater Biol Appl. 2010;30:1014-1021.

21. Yoshimotoa H, Shina Y, Teraia H, Vacantia J. A biodegradable nanofiber scaffold by electrospinning and its potential for bone tissue engineering. Biomaterials. 2003;24:2077-2082.

22. Ma Z, Kotaki M, Yong T, He W, Ramakrishna S. Surface engineering of electrospun polyethylene terephthalate (PET) nanofibers towards development of a new material for blood vessel engineering. Biomaterials. 2005;26:2527-2536.

23. Yang J, Bei J, Wang S. Improving cell affinity of poly (d, 1-Lactide) film modified by anhydrous ammonia plasma treatment. Polym Adv Technol. 2002;13:7.

24. Ryu GH, Yang WS, Roh W, et al. Plasma surface modification of poly (d, 1-lactic-co-glycolic acid) (65/35) film for tissue engineering. Surf Coat Technol. 2005;193:5.

25. Ma R-y, Xiong D-S. Synthesis and properties of physically crosslinked poly (vinyl alcohol) hydrogels. Journal of China University of Mining and Technology. 2008;18:271.

26. Uchino Y, Shimmura S, Miyashita H, et al. Amniotic membrane immobilized poly(vinyl alcohol) hybrid polymer as an artificial cornea scaffold that supports a stratified and differentiated corneal epithelium. J Biomed Mater Res B Appl Biomater. 2007;81:201-206.

27. $\mathrm{Ku} \mathrm{D}$, Braddon L, Wootton D, inventors; Georgia Tech Research Corporation, assignee. Poly(vinyl alcohol) Cryogel. United States patent US 5981826. Nov 9, 1999.

28. Hicks CR, Crawford GJ, Lou X, et al. Corneal replacement using a synthetic hydrogel cornea, AlphaCor ${ }^{\mathrm{TM}}$ : device, preliminary outcomes and complications. Eye. 17:385-392.

29. Jayasuriya AC, Ghosh S, Scheinbeim JI, Lubkin V, Bennett G, Kramer P. A study of piezoelectric and mechanical anisotropies of the human cornea. Biosens Bioelectron. 2003;18:381-387.

30. Ma P, Choi J. Biodegradable polymer scaffolds with well-defined interconnected spherical pore network. Tissue Eng. 2001;1:23-33.

31. Ma Z, Mao Z, Gao C. Surface modification and property analysis of biomedical polymers used for tissue engineering. Colloids Surf B Biointerfaces. 2007;60:137-157.

32. Kim G, Kim W. Highly porous $3 \mathrm{D}$ nanofiber scaffold using an electrospinning technique. J Biomed Mater Res B Appl Biomater. 2007;81B: 104-110.
International Journal of Nanomedicine

\section{Publish your work in this journal}

The International Journal of Nanomedicine is an international, peerreviewed journal focusing on the application of nanotechnology in diagnostics, therapeutics, and drug delivery systems throughout the biomedical field. This journal is indexed on PubMed Central,

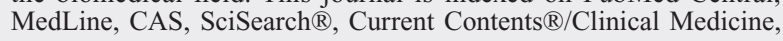

\section{Dovepress}

Journal Citation Reports/Science Edition, EMBase, Scopus and the Elsevier Bibliographic databases. The manuscript management system is completely online and includes a very quick and fair peer-review system, which is all easy to use. Visit http://www.dovepress.com/ testimonials.php to read real quotes from published authors. 